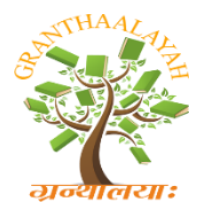

Science

\title{
REDUCTION OF TRUE POWER LOSS BY IMPROVED ALGORITHM
}

\author{
Dr. K. Lenin *1 \\ ${ }^{* 1}$ Professor, Department of EEE, Prasad V.Potluri Siddhartha Institute of Technology, Kanuru, \\ Vijayawada, Andhra Pradesh -520007, India
}

\begin{abstract}
This paper proposes Improved Brain Storm Optimization (IBSO) algorithm is used for solving reactive power problem. predictably, optimization algorithm stimulated by human being inspired problem-solving procedure should be highly developed than the optimization algorithms enthused by collective deeds of ants, bee, etc. In this paper, a new Improved brain storm optimization algorithm defined, which was stimulated by the human brainstorming course of action. In the projected Improved Brain Storm Optimization (IBSO) algorithm, the vibrant clustering strategy is used to perk up the k-means clustering process \& exchange of information wrap all ideas in the clusters to accomplish suitable searching capability. This new approach leads to wonderful results with little computational efforts. In order to evaluate the efficiency of the proposed Improved Brain Storm Optimization (IBSO) algorithm, has been tested standard IEEE 30 bus test system and compared to other standard reported algorithms. Simulation results show that Improved Brain Storm Optimization (IBSO) algorithm is superior to other algorithms in reducing the real power loss.
\end{abstract}

Keywords: Improved Brain Storm Optimization; Optimal Reactive Power; Transmission Loss.

Cite This Article: Dr. K. Lenin. (2018). "REDUCTION OF TRUE POWER LOSS BY IMPROVED ALGORITHM." International Journal of Research - Granthaalayah, 6(8), 105-113. https://doi.org/10.29121/granthaalayah.v6.i8.2018.1404.

\section{Introduction}

Power system constancy is related through security, and it refers to steadiness of service, reliability in frequency and specified voltage restrictions. Main task is to sustain the voltage profiles within the limits by increase or decrease in reactive power. Choosing the ideal parameter of reactive power resources is one of the centre ways for the protected function of transmission structure. The scanty regulation of reactive power sources confines the active power transmission, which can be basis for uncontrolled declined in voltage and tension fall down in the load buses. Optimal reactive power dispatch is one among the main subject for the operation and control of power systems, and it should be carried out properly such that system dependability should not get affected. The gradient method [1, 2], Newton method [3] and linear programming [4-7] experience from the complexity of managing the inequality constraints. In recent times widespread Optimization 
techniques such as genetic algorithms have been proposed to solve the reactive power flow problem [8.9]. In this paper is Improved Brain Storm Optimization (IBSO) Algorithm used to solve the reactive power problem. In the novel Brain Storm Optimization Algorithm (BSO) [10], a k-means clustering method was espoused to group similar data into numerous groups in the converging procedure. In the projected Improved Brain Storm Optimization (IBSO) algorithm, the vibrant clustering strategy is used to perk up the k-means clustering process. The most important view of the vibrant clustering strategy is that; regularly execute the k-means clustering after a definite number of generations, so that the swapping of information wrap all ideas in the clusters to accomplish suitable searching capability. Proposed algorithm has been evaluated in standard IEEE 30 bus test system. Simulation results show that our proposed Improved Brain Storm Optimization (IBSO) approach outperforms all the entitled reported algorithms in minimization of real power loss.

\section{Problem Formulation}

\section{Active power loss}

The objective of the reactive power dispatch is to minimize the active power loss in the transmission network, which can be described as follows:

$F=P L=\sum_{k \in N b r} g_{k}\left(V_{i}^{2}+V_{j}^{2}-2 V_{i} V_{j} \cos \theta_{i j}\right)$

Where $g_{k}$ : is the conductance of branch between nodes $i$ and $j$, Nbr: is the total number of transmission lines in power systems. $\mathrm{P}_{\mathrm{d}}$ : is the total active power demand, $\mathrm{P}_{\text {gi }}$ : is the generator active power of unit $i$, and $\mathrm{P}_{\text {gsalck: }}$ is the generator active power of slack bus.

\section{Voltage Profile Improvement}

For minimizing the voltage deviation in PQ buses, the objective function becomes:

$F=P L+\omega_{v} \times V D$

Where $\omega_{\mathrm{v}}$ : is a weighting factor of voltage deviation.

VD is the voltage deviation given by:

$V D=\sum_{i=1}^{N p q}\left|V_{i}-1\right|$

\section{Equality Constraint}

The equality constraint of the problem is represented by the power balance equation, where the total power generation must cover the total power demand and the power losses:

$P_{G}=P_{D}+P_{L}$

This equation is solved by running Newton Raphson load flow method, by calculating the active power of slack bus to determine active power loss. 


\section{Inequality Constraints}

The inequality constraints reflect the limits on components in the power system as well as the limits created to ensure system security. Upper and lower bounds on the active power of slack bus, and reactive power of generators:

$P_{\text {gslack }}^{\min } \leq P_{\text {gslack }} \leq P_{\text {gslack }}^{\max }$

$Q_{g i}^{\min } \leq Q_{g i} \leq Q_{g i}^{\max }, i \in N_{g}$

Upper and lower bounds on the bus voltage magnitudes:

$V_{i}^{\min } \leq V_{i} \leq V_{i}^{\max }, i \in N$

Upper and lower bounds on the transformers tap ratios:

$T_{i}^{\min } \leq T_{i} \leq T_{i}^{\max }, i \in N_{T}$

Upper and lower bounds on the compensators reactive powers:

$Q_{c}^{\min } \leq Q_{c} \leq Q_{C}^{\max }, i \in N_{C}$

Where $\mathrm{N}$ is the total number of buses, $\mathrm{N}_{\mathrm{T}}$ is the total number of Transformers; $\mathrm{N}_{\mathrm{c}}$ is the total number of shunt reactive compensators.

\section{Brain Storm Optimization Algorithm (BSO)}

We all have experienced that, when we face a difficult problem which, single person can't solve, a group of persons, particularly with dissimilar environment, get together to brain storm and the problem can frequently be solved with high possibility. Enormous and un-expectable intelligence can happen from interactive teamwork of human beings. One way to help human beings to interactively team up to generate great ideas is to acquire jointly a group of people to brainstorm. A brainstorming procedure normally described as follows,

Rule 1. Acquire jointly a brainstorming group of people with dissimilar background as feasible.

Rule 2. Generate many ideas.

Rule 3. Contain a number of, like 4 or 5 , clientele perform as the owners of the problem. To pick up a number of i.e. one from every owner, information as superior ideas for solving the problem.

Rule 4. Use the ideas selected up in the rule 3 with superior possibility than other information as clues, and produce more ideas.

Rule 5. Encompass the owners to choose up several better ideas generated as did in rule 3.

Rule 6. Arbitrarily choose an object and make use of the functions and appearance of the object as clues, \& produce more ideas.

Rule 7. Enclose the owners to pick up several healthier ideas;

Rule 8 . Optimistically a high-quality solution can be obtained by taking into account or by merging the ideas generated. 
In a brainstorming procedure, usually there are a catalyst, a brainstorming cluster of people, and numerous owners of the problem to be solved. The role of the catalyst is to facilitate the idea production (brainstorming) procedure by enforcing the brainstorming cluster to obey the Osborn's $[11,12]$ original four rules of idea generation in a brainstorming procedure. The catalyst should not be caught up in generating ideas itself, but facilitate the brainstorming procedure only. The rule for selecting catalyst or facilitator is to have a catalyst to have facilitation knowledge, but have less proficiency on the background knowledge connected to the problem to be solved as possible. The purpose of this is to have generated ideas to have less, if not nothing, then biases from the catalyst.

Osborn's Original Rules for Idea Generation in a Brainstorming Process are as follows,

Rule 1. Defer verdict

Rule 2. Everything Goes

Rule 3. Cross-fertilize

Rule 4. Go for capacity

- Rule 1 says that there is no idea can be seen as bad idea. All ideas are high-quality ideas only. It is ill-advised to judge whether a projected idea is a high-quality or dreadful idea. Any decision or censure must be held back until at least the conclusion of the brainstorming process.

- Rule2 says that everything coming to your mind in the period of the brainstorming process is an idea merit to be pooled and recorded. Don't let any idea or contemplation mistreated.

- Rule 3 says that bunch of ideas be capable and should be based on ideas previously generated. Every generated idea be capable and should dish up as a clue to generate more ideas.

- Rule 4 says that it is essential to generate lot of ideas as possible. We primary go for amount of generated ideas. The superiority will come from quantity obviously. Lacking in generating large quantity of ideas, it is difficult or impossible, to come out ideas with highquality.

\section{Brain Storm Optimization Algorithm (BSO)}

Step 1. Arbitrarily produce n potential solutions (individuals);

Step 2. Huddle $\mathrm{n}$ individuals into m clusters;

Step 3. Calculate the $\mathrm{n}$ individuals;

Step 4. Grade the individuals in every cluster and document the best individual as cluster centre in each cluster;

Step 5. Arbitrarily generate a value between 0 and 1;

a) If the value is smaller than a predestined probability then,

i. Arbitrarily select a cluster centre;

ii. Arbitrarily generate an individual to substitute the selected cluster centre;

Step 6. Create new individuals, 
$X_{\text {new }}^{d}=X_{\text {selected }}^{d}+\xi * n(\mu, \sigma)$

where $X_{\text {selected }}^{d}$ is the $\mathrm{d}^{\text {th }}$ dimension of the individual selected to generate new individual; $X_{\text {new }}^{d}$ is the $\mathrm{d}^{\text {th }}$ dimension of the individual newly generated; $\mathrm{n}(\mu, \sigma)$ is the Gaussian random function with mean $\mu$ and variance $\sigma$; the $\xi$ is a coefficient that weights the contribution of the Gaussian random value. $\xi$ can be calculated as

$\xi=\operatorname{logsig}\left(\left(0.5 * \max _{-}\right.\right.$iteration - current_iteration $\left.) / k\right) *$ rand ()

Where logsig () is a logarithmic sigmoid transfer function, max_iteration is the maximum number of iterations, and current_iteration is the current iteration number, $\mathrm{k}$ is for changing logsig () function's slope, and rand () is a random value within $(0,1)$.

a) Arbitrarily generate a value between 0 and 1 ;

b) If the value is less than a probability then,

i. Arbitrarily select a cluster with a probability;

ii. Create a random value between 0 and 1 ;

iii. If the value is smaller than a predestined probability then,

1) Choose the cluster centre and include random values to it to create new individual.

iv.Or else arbitrarily select an individual from this cluster and include random value to the individual to create new individual.

c)Or else arbitrarily select two clusters to create new individual

i. Generate a random value;

ii. If it is less than a predestined probability, then the two cluster centres are pooled together and then added with random values to create new individual;

iii. Or else, two individuals from every selected cluster are arbitrarily selected has to be combined and added with random values to create new individual.

d) The newly created individual is compared with the lively individual with the same individual catalogue. The superior one is kept and recorded as the new individual.

Step 7. If $\mathrm{n}$ new individuals have been created, then go to step 8 or else go to step 6;

Step 8. Conclude if predestined maximum number of iterations has been reached or else go to step 2.

\section{Improved Brain Storm Optimization (IBSO) Algorithm by Vibrant Clustering Approach}

In the novel Brain Storm Optimization Algorithm (BSO), a k-means clustering method was espoused to group similar data into numerous groups in the converging procedure. As we all known, the k-means clustering technique is time consuming and requires large time for the computation process. Throughout the evolutionary procedure, BSO carry out the k-means clustering in every generation to cluster ideas. Conversely, it is not essential to use k-means clustering technique to assemblage the ideas into dissimilar groups in every generation. In the projected Improved Brain Storm Optimization (IBSO) algorithm, the vibrant clustering strategy is used to perk up the k-means clustering process. The most important view of the vibrant clustering 
strategy is that; regularly execute the k-means clustering after a definite number of generations, so that the swapping of information wrap all ideas in the clusters to accomplish suitable searching capability. Therefore, in our vibrant clustering stratagem, the important point is the size of reclustering phase. If the re-clustering phase is lofty, the algorithm will get an elevated degree of exploitation and a soaring convergence rate, and the time difficulty of the algorithm will be respectively higher. Conversely, if the re-clustering period is inferior, the algorithm will attain more exploration and diversity, and the run time of the algorithm will be condensed in percentage. So, a suitable re-clustering phase helps to balance the exploitation and exploration of the algorithm, and will attain improved performance than the original BSO algorithm. In this paper, we use a probabilistic parameter p_vibrant to denote the re-clustering period. p_vibrant is a value between 0 to 1.

\title{
Algorithm for the implementation of vibrant clustering strategy
}

\author{
Vibrant Clustering \\ Commence \\ If rand ()$<P_{-}$vibrant \\ Implement $\mathrm{k}$-means process \\ End \\ End
}

To regulate the convergence speed as the evolution goes in idea generation, the original BSO algorithm defines a fine-tune factor. Through numerical research, we find that at first the fine-tune factor keeps around 1, while when part the number of generations has been attained, it quickly turns to near 0 . This technique to control the size of step can also balance exploration and exploitation at different penetrating generations. Conversely, it just takes effect only for very small interval. Therefore, we bring in a simple vibrant step size stratagem. The vibrant function is described as follows,

$\xi=$ random $^{*} \exp \left(1-\frac{\text { maximum_iteration }_{\text {maximum }}}{\text { iteration }- \text { current }_{\text {iteration }}+1}\right)$

Where random is an arbitrary value between 0 and 1. The max_iteration and current_iteration also indicate the maximum number of iterations and current number of iteration, correspondingly.

Commence

Arbitrarily initialize $\mathrm{N}$ ideas and estimate their fitness

Set up m cluster centers $(m<N)$

While (end condition not met)

Implement: vibrant Clustering ( )

For ( $\mathrm{i}=1$ to $\mathrm{N}$ )

If random ()$<P \_o n e$

Arbitrarily choose a cluster

If random ()$<P \_o n e \_c e n t e r$

Provide the cluster center plan to $\mathrm{X}_{\text {selected }}$

Else

Arbitrarily choose a notion in a cluster to $\mathrm{X}_{\text {selected }}$ 
End if

Else

Arbitrarily choose two clusters

If random ()$<P_{-} t w o \_c e n t e r$

Merge the two clusters' center notions to $\mathrm{X}_{\text {selected }}$

Else

Merge two random notions to $\mathrm{X}_{\text {selected }}$

End if

End if

Generate $X_{\text {new }}$ using $X_{\text {selected }}$ with formula (10) and (12)

Recognize $X_{\text {new }}$ if $f\left(X_{\text {new }}\right)$ is superior than $f\left(X_{i}\right)$

End for

End while

End

\section{Simulation Results}

Improved Brain Storm Optimization (IBSO) algorithm validity has been verified by testing in standard IEEE 30-bus without considering Voltage stability evaluation. Standard IEEE 30-bus has 41 branches, 6 generator-bus, 4 transformer-tap settings, with 2 shunt reactive compensators buses. 2, 5, 8, 11 and 13 are considered as PV generator buses and Bus 1 is taken as slack bus, others are PQ load buses. In Table 1 Control variables limits are given.

Table 1: Primary Variable Limits (PU)

\begin{tabular}{|c|c|c|c|}
\hline Name of the Variables & Minimum & Maximum & Class \\
\hline Generator Bus & 0.95 & 1.1 & Continuous \\
\hline Load Bus & 0.95 & 1.05 & Continuous \\
\hline Transformer-Tap & 0.9 & 1.1 & Discrete \\
\hline Shunt Reactive Compensator & -0.11 & 0.31 & Discrete \\
\hline
\end{tabular}

In Table 2 gives the power limits of generators buses.

Table 2: Generators Power Limits

\begin{tabular}{|l|l|l|l|l|l|}
\hline Bus number & Pg & Pgminimum & Pgmaximum & Qgminimum & Qgmaximum \\
\hline 1 & 96.00 & 49 & 200 & 0 & 10 \\
\hline 2 & 79.00 & 18 & 79 & -40 & 50 \\
\hline 5 & 49.00 & 14 & 49 & -40 & 40 \\
\hline 8 & 21.00 & 11 & 31 & -10 & 40 \\
\hline 11 & 21.00 & 11 & 28 & -6 & 24 \\
\hline 13 & 21.00 & 11 & 39 & -6 & 24 \\
\hline
\end{tabular}

Table 3 shows the values of control variables. Table 4 narrates the performance of the proposed algorithm. Voltage deviations during the iterations are given in Fig 1 and overall comparison of the results of optimal solution obtained by various methods is given in Table 5. 
Table 3: After optimization values of control variables

\begin{tabular}{|l|l|}
\hline List of Control Variables & IBSO \\
\hline V1 & 1.04120 \\
\hline V2 & 1.04100 \\
\hline V5 & 1.01810 \\
\hline V8 & 1.02720 \\
\hline V11 & 1.06810 \\
\hline V13 & 1.04800 \\
\hline T4,12 & 0.000 \\
\hline T6,9 & 0.010 \\
\hline T6,10 & 0.900 \\
\hline T28,27 & 0.910 \\
\hline Q10 & 0.100 \\
\hline Q24 & 0.100 \\
\hline Real power loss & 4.2382 \\
\hline Voltage deviation & 0.9080 \\
\hline
\end{tabular}

Table 4: Performance of IBSO algorithm

\begin{tabular}{|l|l|}
\hline No. of Iterations & 34 \\
\hline Time taken (secs) & 10.04 \\
\hline Real power loss (MW) & 4.2382 \\
\hline
\end{tabular}

Table 5: Comparison of results

\begin{tabular}{|l|l|}
\hline Method & Real power loss (MW) \\
\hline SGA (Wu et al., 1998) & 4.98 \\
\hline PSO (Zhao et al., 2005) & 4.9262 \\
\hline LP (Mahadevan et al., 2010) & 5.988 \\
\hline EP (Mahadevan et al., 2010) & 4.963 \\
\hline CGA (Mahadevan et al., 2010) & 4.980 \\
\hline AGA (Mahadevan et al., 2010) & 4.926 \\
\hline CLPSO (Mahadevan et al., 2010) & 4.7208 \\
\hline HSA (Khazali et al., 2011) & 4.7624 \\
\hline BB-BC (Sakthivel et al., 2013) & 4.690 \\
\hline MCS(Tejaswini sharma et al.,2016) & 4.87231 \\
\hline Proposed IBSO & 4.2382 \\
\hline
\end{tabular}

\section{Conclusion}

In this paper Improved Brain Storm Optimization (IBSO) algorithm is used to solve 1 reactive power problem by including various generator constraints. In the projected Improved Brain Storm Optimization (IBSO) algorithm, the vibrant clustering strategy is used to perk up the k-means clustering process. Efficiency of the proposed Improved Brain Storm Optimization (IBSO) algorithm has been demonstrated on standard IEEE 30 bus test system. Simulation results shows that Real power loss has been significantly reduced and voltage profile index within the particular limits. 


\section{References}

[1] O. Alsac, and B. Scott, "Optimal load flow with steady state security", IEEE Transaction. PAS 1973, pp. 745-751.

[2] Lee K Y, Paru Y M, Oritz J L -A united approach to optimal real and reactive power dispatch, IEEE Transactions on power Apparatus and systems 1985: PAS-104: 1147-1153

[3] A. Monticelli, M. V.F Pereira, and S. Granville, "Security constrained optimal power flow with post contingency corrective rescheduling", IEEE Transactions on Power Systems: PWRS-2, No. 1, pp.175-182.,1987.

[4] Deeb N, Shahidehpur S.M, Linear reactive power optimization in a large power network using the decomposition approach. IEEE Transactions on power system 1990: 5(2): 428-435

[5] E. Hobson, 'Network consrained reactive power control using linear programming, 'IEEE Transactions on power systems PAS -99 (4), pp 868-877, 1980

[6] K.Y Lee, Y.M Park, and J.L Oritz, "Fuel -cost optimization for both real and reactive power dispatches", IEE Proc; 131C, (3), pp.85-93.

[7] M.K. Mangoli, and K.Y. Lee, "Optimal real and reactive power control using linear programming", Electr.Power Syst.Res, Vol.26, pp.1-10,1993.

[8] K.Anburaja, "Optimal power flow using refined genetic algorithm", Electr.Power Compon.Syst , Vol. 30, 1055-1063,2002.

[9] D. Devaraj, and B. Yeganarayana, "Genetic algorithm based optimal power flow for security enhancement", IEE proc-Generation. Transmission and. Distribution; 152, 6 November 2005.

[10] Yuhui Shi Xi'an Jiaotong, Brain Storm Optimization Algorithm, Y. Tan et al. (Eds.): ICSI 2011, Part I, LNCS 6728, pp. 303-309, 2011.

[11] Smith, R.: The 7 Levels of Change, 2nd edn. Tapeslry Press (2002).

[12] Zijian Cao, Xiaofeng Rong, Zhiqiang Du, "An Improved Brain Storm Optimization with Dynamic Clustering Strategy”, MATEC Web of Conferences 95, 19002 (2017), ICMME 2016.

[13] Wu.Q.H,Y.J.Cao, and J.Y.Wen,(1998), "Optimal reactive power dispatch using an adaptive genetic algorithm”, Int.J.Elect.Power Energy Syst. Vol 20. Pp. 563-569.

[14] Zhao.B,C.X.Guo,andY.J.CAO,(2005),"Multiagent-based particle swarm optimization approach for optimal reactive power dispatch", IEEE Trans. Power Syst. Vol. 20, no. 2, pp. 1070-1078.

[15] Mahadevan.K,KannanP.S,(2010)"Comprehensive Learning Particle Swarm Optimization for Reactive Power Dispatch”, Applied Soft Computing, Vol. 10, No. 2, pp. 641-52.

[16] Khazali.A.H,M.Kalantar,(2011),“Optimal Reactive Power Dispatch based on Harmony Search Algorithm", Electrical Power and Energy Systems, Vol. 33, No. 3, pp. 684-692.

[17] Sakthivel.S,M.Gayathri,V.Manimozhi,(2013),"A Nature Inspired Optimization Algorithm for Reactive Power Control in a Power System”, International Journal of Recent Technology and Engineering, pp29-33Vol.2,Issue-1.

[18] Tejaswini Sharma,Laxmi Srivastava,Shishir Dixit (2016). "Modified Cuckoo Search Algorithm For Optimal Reactive Power Dispatch", Proceedings of 38 th IRF International Conference, pp48. 20th March, 2016, Chennai, India, ISBN: 978-93-85973-76-5

*Corresponding author.

E-mail address: gklenin@ gmail.com 